\title{
Prediction of the Stribeck curve under full-film Elastohydrodynamic Lubrication
}

\author{
Y. Zhang ${ }^{\mathrm{a}}$, N. Biboulet ${ }^{\mathrm{a}}$, C.H. Venner ${ }^{\mathrm{b}}$, A.A. Lubrecht ${ }^{\mathrm{a}, *}$ \\ ${ }^{a}$ Univ Lyon, INSA-Lyon, CNRS UMR5259, LaMCoS, F-69621, France \\ ${ }^{\mathrm{b}}$ Faculty of Mechanical Engineering, University of Twente, 7500 AE, Enschede, the Netherlands
}

\section{A R T I C L E I N F O}

\section{Keywords:}

Stribeck curve

Full-film EHL

Numerical simulation

Piezoviscous elastic regime

Amplitude Reduction Theory

\begin{abstract}
A B S T R A C T
The Stribeck curve shows the friction coefficient as a function of speed, viscosity and load. The viscosity times speed over load parameter can be interpreted as a film thickness. The film thickness over roughness parameter unifies friction curves in the isoviscous rigid regime. In this paper, the Stribeck curve is predicted numerically in the full-film Elastohydrodynamic Lubrication regime. It is shown that the lambda ratio is not the most appropriate parameter. A more elaborate parameter including the operating conditions and based on the Amplitude Reduction Theory [1] gives much better results. For a complex surface topography, the full numerical simulation is time-consuming. A rapid prediction method is proposed. Good agreement is found between the full numerical simulation and the prediction.
\end{abstract}

\section{Introduction}

Most machine elements are working under elastohydrodynamically lubricated (EHL) conditions. Understanding the frictional behavior in such contacts play an important role for reducing friction, preventing wear as well as improving service life. The Stribeck curve: friction coefficient as a function of the Sommerfeld number, is a useful tool to describe the frictional characteristics of a liquid lubricant [2]. In 1879, Thurston [3] gave precise values of the friction coefficient and he was probably the first person to report that the friction coefficient passed through a minimum as the load increased [4]. Twenty years later, Stribeck [5] published results of a carefully conducted and wide-ranging series of experiments on journal bearings, which are frequently referred to as 'the Stribeck curve'. Gümbel [6] analysed Stribeck's experimental results in a single curve by plotting the friction against the parameter $\eta \omega / \bar{p}$, where $\eta$ is the lubricant viscosity, $\omega$ is the angular velocity of the shaft and $\bar{p}$ is the load per unit length. At the same time, Hersey [2] conducted experiments on journal bearings and plotted the friction coefficient against the load, speed, temperature, viscosity and rate of oil supply. He showed that hydrodynamic friction should be a function of $\eta n / p$ in which $n$ is the rotational speed and $p$ is the pressure. Many years later, Wilson and Barnard [7] replotted the Stribeck curve by introducing a new variable i.e. $z n / p$, where the lower-case $z$ stands for the lubricant viscosity. Subsequently, McKee [8] provided a similar dimensionless group $Z N / P$. Vogelpohl et al. [9] incorporated the boundary and fluid friction coefficient and showed a transition from the hydrodynamic lubrication regime to the mixed lubrication regime. All of the work mentioned above is performed under low pressure conditions, in the isoviscous rigid regime.

The situation for non-conforming contacts, such as those occurring in rolling element bearings, gears and seals, is somewhat different [10]. Shotter [11] experimentally showed that the friction increases with the surface roughness. Tallian and his co-workers $[12,13]$ proposed a ratio $\left|\xi_{0}\right|$ between the elastohydrodynamic film thickness and the composite root mean square roughness to represent the mixed elastodydrodynamic regime $\left(1<\left|\xi_{0}\right|<4\right)$. Poon [14] was concerned with the transition from the boundary to the mixed regime with a dimensionless parameter $1.0 \leq \xi \leq 2.0$ and the transition from mixed to full EHL region with $2.0<\xi \leq 2.4$ by using electrical-conductivity measurements. Bair and Winer [15] plotted the reduced traction coefficient as a function of a lambda ratio by performing sliding-rolling experiments. They found that when the lambda ratios is less than 2 the contact moves into the mixed regime. In general, the Stribeck curve is plotted versus the lambda ratio, which is defined as central film thickness to composite surface roughness. Typically, the lubrication regimes can be divided as [16]: $\lambda>3.0$ represents the full-film regime, $1.0 \leq \lambda \leq 3.0$ is the mixed EHL regime and $\lambda<1.0$ indicates the boundary regime. However, study [17] shows that this lambda ratio is not a suitable parameter to determine lubrication states when some aspects such as non-Newtonian, thermal and transient effects are considered. Transition

\footnotetext{
* Corresponding author.

E-mail address: ton.lubrecht@insa-lyon.fr (A.A. Lubrecht).
} 


\begin{tabular}{|c|c|}
\hline \multicolumn{2}{|c|}{ Notation } \\
\hline$a_{d}$ & deformed amplitude in the center of the contact [m] \\
\hline$A_{d}$ & $\begin{array}{l}\text { dimensionless deformed amplitude in the center of the } \\
\text { contact }\end{array}$ \\
\hline$a_{i}$ & initial amplitude $[\mathrm{m}]$ \\
\hline$A_{i}$ & dimensionless initial amplitude \\
\hline$a_{h}$ & the radius of the contact area $a_{h}=\sqrt[3]{3 w R_{x} /\left(2 E^{\prime}\right)}$ [m] \\
\hline$E^{\prime}$ & $\begin{array}{l}\text { reduced modulus of elasticity } 2 / E^{\prime}=\left(1-v_{1}^{2}\right) / E_{1}+ \\
\left(1-v_{2}^{2}\right) / E_{2}[\mathrm{~Pa}]\end{array}$ \\
\hline$f$ & $\begin{array}{l}\text { the friction force induced by the shearing of the lubricant } \\
{[N]}\end{array}$ \\
\hline$F$ & the dimensionless friction force \\
\hline$G$ & dimensionless material parameter $G=\alpha E^{\prime}$ \\
\hline$h$ & film thickness $[\mathrm{m}]$ \\
\hline$H$ & dimensionless film thickness $H=h R_{x} / a_{h}^{2}$ \\
\hline$h_{c}$ & central film thickness $[\mathrm{m}]$ \\
\hline$H_{c}$ & $\begin{array}{l}\text { dimensionless central film thickness for smooth case } \\
H_{c}=h_{c} R_{x} / a_{h}^{2}\end{array}$ \\
\hline$H_{x}, H_{y}$ & dimensionless mesh sizes in $\mathrm{x}$ and $\mathrm{y}$ directions respectively \\
\hline$L_{x}, L_{y}$ & lengths of final topography $[\mathrm{m}]$ \\
\hline$L$ & dimensionless material parameter (Moes) $L=G(2 U)^{0.25}$ \\
\hline$M$ & 2d dimensionless load parameter (Moes) $M=W_{2}(2 U)^{-0.75}$ \\
\hline$p$ & pressure $[\mathrm{Pa}]$ \\
\hline $\begin{array}{l}p_{s} \\
\delta p\end{array}$ & $\begin{array}{l}\text { Pressure for smooth cases }[\mathrm{Pa}] \\
\text { pressure fluctuations }[\mathrm{Pa}]\end{array}$ \\
\hline$\Delta P$ & dimensionless pressure fluctuations $\Delta P=\delta p / p_{h}$ \\
\hline $\begin{array}{l}q_{x}, q_{y} \\
R_{x}\end{array}$ & $\begin{array}{l}\text { Wavenumbers in } \mathrm{x} \text { and } \mathrm{y} \text { directions respectively }[1 / \mathrm{m}] \\
\text { reduced radius of curvature in } \mathrm{x}: 1 / R_{x}=1 / R_{1 x}+1 / R_{2 x}[\mathrm{~m}]\end{array}$ \\
\hline$R_{y}$ & reduced radius of curvature in y: $R_{y}=R_{x}[\mathrm{~m}]$ \\
\hline$r r$ & surface roughness $[\mathrm{m}]$ \\
\hline$r r^{d}$ & Deformed surface roughness $[\mathrm{m}]$ \\
\hline$R R$ & dimensionless surface roughness $R R=r r \cdot R_{x} / a_{h}$ \\
\hline$t$ & time $[\mathrm{s}]$ \\
\hline
\end{tabular}

$T$

$\bar{u}$

$\delta u$

$U$

$\Delta U$

$U_{\text {rat }}$

$w$

$W_{2}$

$x$

$X$

$\bar{X}$

$y$

Y

$\alpha$

$\bar{\alpha}$

$\nabla_{2}$

$\bar{\lambda}$

$\lambda_{x}, \lambda_{y}$

$\overline{\bar{\lambda}}$

$\eta$

$\eta_{0}$

$\bar{\eta}$

$\rho$

$\rho_{0}$

$\bar{\rho}$

subscripts

$a, b \quad$ inlet, outlet

$i, d \quad$ initial, deformed

$r, s \quad$ rough, smooth

st start

normal load $[\mathrm{N}]$

viscosity [Pa:s]

density $\left[\mathrm{Kg} \cdot \mathrm{m}^{-3}\right]$ dimensionless time $T=t \bar{u} / a_{h}$

mean velocity $\bar{u}=\left(u_{1}+u_{2}\right) / 2[\mathrm{~m} / \mathrm{s}]$

sliding speed $\delta u=u_{1}-u_{2}[\mathrm{~m} / \mathrm{s}]$

dimensionless speed parameter $U=\left(\eta_{0} \bar{u}\right) /\left(E^{\prime} R_{x}\right)$

slide-to-roll ratio $\Delta U=\delta u / \bar{u}=\left(u_{1}-u_{2}\right) / \bar{u}$

slip parameter $U_{r a t}=u_{1} / \bar{u}$

2d dimensionless load parameter $W_{2}=w /\left(E^{\prime} R_{x}^{2}\right)$

coordinate in the rolling direction $[\mathrm{m}]$

dimensionless coordinate $X=x / a_{h}$

dimensionless surface feature location

coordinate perpendicular to $x[\mathrm{~m}]$

dimensionless coordinate $Y=y / a_{h}$

pressure viscosity index $[1 / \mathrm{Pa}]$

dimensionless viscosity index $\bar{\alpha}=\alpha p_{h}$

shear stress induced by the shearing of the lubricant $[\mathrm{Pa}]$

dimensionless wavelength parameter defined in Ref. [1]

dimensionless speed parameter

wavelength in $\mathrm{x}$, $\mathrm{y}$ direction, $\lambda_{x}=\lambda_{y}=\lambda[\mathrm{m}]$

dimensionless wavelength $\overline{\bar{\lambda}}=\lambda / a_{h}$

the atmospheric viscosity [Pa-s]

dimensionless viscosity $\bar{\eta}=\eta / \eta_{0}$

the atmospheric density $\left[\mathrm{Kg} \cdot \mathrm{m}^{-3}\right]$

dimensionless density $\bar{\rho}=\rho / \rho_{0}$ locations from mixed to boundary lubrication regime or from full-film to mixed lubrication regime are still ambiguous. Therefore, an appropriate grouping including the speed, film thickness and roughness is required. Schipper [18] suggested a so-called Lubrication number $L^{\prime}$, which takes viscosity, speed and pressure into consideration, to detect the variation of the friction coefficient.

Because the full numerical model of mixed lubrication is complicated and the knowledge of the physico-chemical interactions in boundary lubrication is still insufficient, most of the work was done experimentally. Recently, Gelinck [19] extended Johnson's model [20] to calculate the coefficient of friction for the whole mixed EHL regime. Lu and Khonsari [21] examined the behavior of the Stribeck curve theoretically and experimentally on a journal bearing and found a good agreement. Wang et al. [22] presented a numerical approach developed on the basis of deterministic solutions of mixed lubrication to evaluate sliding friction. Meanwhile, they measured the sliding friction on a commercial test rig. Both results were plotted against sliding velocities and also showed good agreement. Kalin [23] investigated changes of the Stribeck curve when one or two surfaces in the contact are non-fully wetted. Afterwards, Kalin [24] tested the variations of the friction coefficient with diamond-like carbon coatings (DLC). Results showed that in the boundary-lubrication regime the Stribeck curve of the DLC contacts has an inverse shape to that of the steel contacts. Zhang [25] developed a numerical approach assuming the asperity interaction friction is proportional to the contact area to predict the mixed EHL friction coefficient. Bonaventure [26] and his co-authors conducted rolling-sliding experiments with randomly surface roughness, they found that the onset of ML at a higher entrainment products $\eta_{0} u_{e}$ (in which $\eta_{0}$ is inlet viscosity and $u_{e}$ is entrainment speed) and a relevant roughness scalar parameter was obtained to predict the onset position. In previous work on the Stribeck curve, the friction coefficient is depicted as a function of the oil film thickness to the combined surface roughness. Recent work [1] shows that under very high pressure situations, surface roughness will be deformed, and this deformation depends on the operating conditions. Hence, the old parameter "lambda ratio" is replaced by a new parameter. The current study employs the Amplitude Reduction Theory [1] to predict the Stribeck curve numerically in the full-film EHL regime. A new parameter including the operating conditions is derived to unify all simulation results into a single curve. Meanwhile, a rapid prediction method based on the roughness power spectral density (PSD) is provided to predict the relative friction increase due to roughness which is shown to yield results with good engineering accuracy for practical use.

\section{EHL model}

\subsection{Equations}

The classical Reynolds equation for the transient case reads [27]:

$\frac{\partial}{\partial x}\left(\frac{\rho h^{3}}{12 \eta} \frac{\partial p}{\partial x}\right)+\frac{\partial}{\partial y}\left(\frac{\rho h^{3}}{12 \eta} \frac{\partial p}{\partial y}\right)-\bar{u} \frac{\partial(\rho h)}{\partial x}-\frac{\partial(\rho h)}{\partial t}=0$

with $p=0$ on the boundaries and the cavitation condition $p \geq 0$ everywhere. Where $p$ represents the pressure, $h$ denotes the film thickness and $\bar{u}=\left(u_{1}+u_{2}\right) / 2$ is the mean velocity.

The density $\rho$ and the viscosity $\eta$ are defined by the Dowson and Higginson relation [28] and the Roelands viscosity pressure relation [29] respectively. Meanwhile the film thickness equation is denoted as: 
$h(x, y, t)=h_{0}(t)+\frac{x^{2}}{2 R_{x}}+\frac{y^{2}}{2 R_{y}}-r r(x, y, t)+\frac{2}{\pi E^{\prime}} \int_{-\infty}^{+\infty} \int_{-\infty}^{+\infty} \frac{p\left(x^{\prime}, y^{\prime}, t\right) d x d y}{\sqrt{\left(x-x^{\prime}\right)^{2}+\left(y-y^{\prime}\right)^{2}}}$

where

$2 / E^{\prime}=\left(1-v_{1}^{2}\right) / E_{1}+\left(1-v_{2}^{2}\right) / E_{2}$

and $v$ is the Poisson ratio, and $E$ is the elastic modulus of the two bodies 1 and 2 respectively. $E^{\prime}$ is called the reduced elastic modulus and $\operatorname{rr}(x, y, t)$ stands for the undeformed roughness of the two surfaces.

Finally, the force balance equation should be satisfied all the time.

$w(t)=\int_{-\infty}^{+\infty} \int_{-\infty}^{+\infty} p\left(x^{\prime}, y^{\prime}, t\right) d x^{\prime} d y^{\prime}$

where $w$ is the normal load.

In the full-film EHL regime, the friction force is mainly determined by the shearing of the lubricant in the contact zone, which is written as

$f(t)=\iint \tau(x, y, t) d x d y=\iint \eta(p, t) \frac{\delta u}{h(x, y, t)} d x d y$

where $\delta u=u_{1}-u_{2}$ is the sliding speed, $f$ and are friction force and shear stress respectively.

In the current work, a Newtonian lubricant, an isothermal regime and a circular contact condition are proposed. To reduce the number of the independent parameters, we introduce $P=p / p_{h}, X=x / a_{h}, Y=y / a_{h}$ and $H=h R_{x} / a_{h}^{2}$, based on Hertz and $T=t \bar{u} / a_{h}, \bar{\eta}=\eta / \eta_{0}$ and $\bar{\rho}=\rho / \rho_{0}$. Dimensionless forms of equations (1)-(3) are used as expressed in Ref. [27].

The dimensionless form of Eq. (4) is

$F(T)=\iint \bar{\eta}(P, T) \frac{\Delta U}{H(X, Y, T)} d X d Y$

in which $\Delta U=\delta u / \bar{u}$ is the slide/roll ratio.

Consequently, the relative friction coefficient is defined by

$\frac{\mu_{r}}{\mu_{s}}(T)=\frac{F_{r}(T)}{w} / \frac{F_{s}}{w}=\frac{F_{r}(T)}{F_{s}}$

where the subscripts $r$ and $s$ stand for the rough and smooth case respectively.

\subsection{Numerical solution}

In order to solve the EHL contact model, the well-known Multigrid technique [27] is used. However, the performance of the existing MultiGrid algorithm in terms of efficiency and stability deteriorates when large variations of the coefficient $\rho h^{3} / \eta$ occur on a small scale, as in the case for very rough surfaces. An efficient way of restoring the performance is by constructing the coarse grid operator, and the intergrid transfers as proposed by Alcouffe et al. [30]. The partial differential equation considered by Alcouffe is

$$
\begin{aligned}
- & \nabla \cdot(D(X, Y, T) \nabla U(X, Y, T))+\sigma(X, Y, T) U(X, Y, T) \\
& =F F(X, Y, T) \quad(X, Y) \in \Omega
\end{aligned}
$$

where $D, U$ and $F F$ are discontinuous functions on the bounded region $\Omega$.

The dimensionless Reynolds equation has the same form as Eq. (7) with: $U=P, \sigma=0, D=-\bar{\rho} H^{3} / \bar{\eta} \bar{\lambda}$ and $F F=\partial(\bar{\rho} H) / \partial X+\partial(\bar{\rho} H) / \partial T$. In this study, the calculation domain $\Omega$ is a rectangle $\left[X_{a}, X_{b}\right] \times\left[Y_{-a}, Y_{a}\right]$ covered by a uniform grid. The mesh size in the two directions is $H x=\left(X_{b}-X_{a}\right) / N x$ and $H y=\left(Y_{a}-Y_{-a}\right) / N y$ respectively, in which $N_{x}$ and $N_{y}$ are the number of grid points in $\mathrm{x}$ - and y-direction. According to Eq. [2.4] in Ref. [30], the dimensionless Reynolds equation is discretized as:

$$
\begin{aligned}
& A_{i, j, k}\left(P_{i, j+1, k}-P_{i, j, k}\right)+A_{i, j-1, k}\left(P_{i, j-1, k}-P_{i, j, k}\right)+B_{i, j, k}\left(P_{i+1, j, k}-P_{i, j, k}\right) \\
& \quad+B_{i, j-1, k}\left(P_{i-1, j, k}-P_{i, j, k}\right)=f f_{i, j, k}
\end{aligned}
$$

where $A_{i, j, k}=-0.5\left(D_{i, j, k}+D_{i, j+1, k}\right), \quad B_{i, j, k}=-0.5\left(D_{i, j, k}+D_{i+1, j, k}\right)$, and $f f_{i, j, k}=(H x \cdot H y) F F_{i, j, k}$. The right hand side of Reynolds equation $f f_{i, j, k}$ is discretized using a second order backward discretization.

Compared to the classical Multilevel method, improvements referred in Ref. [30] are mainly represented in two aspects. One is constructing new intergrid transfer operators $I_{H}^{h}$ and $I_{h}^{H}$ based on coefficients $A_{i, j, k}$ and $B_{i, j, k}$, which allows $D \nabla P$ to be continuous over the whole calculation domain and gives a more reasonable physical representation on a coarse grid. Another is rebuilding the coarse grid operator using Galerkin coarsing $L^{H}=I_{h}^{H} L^{h} I_{H}^{h}$ to form a good approximation to the fine grid operator to eliminate low frequency error components.

\section{PSD relative friction model}

An alternative approach to predict the relative friction coefficient for a complex rough surface is by applying the power spectral density. The power spectral density (PSD) is a mathematical tool that can decompose a rough surface into harmonic components of different frequencies [31], which enables the pressure increase to be calculated analytically for each frequency component. Subsequently, the shear stress for the whole rough surface can be obtained. At last, the relative friction coefficient is obtained. The calculation process is as follows:

A rough surface topography $r r_{x, y}$ can be expressed in the frequency domain by means of the Fourier transform:

$r r_{q_{x}, q_{y}}=\frac{4}{N_{x} N_{y}} \sum_{x, y}\left(r r_{x, y}\right) e^{-i\left(q_{x} x+q_{y} y\right)}$

where $r r_{x, y}$ is the discrete form of the surface roughness $\operatorname{rr}(x, y), q_{x}$ and $q_{y}$ are the wavenumbers in $\mathrm{x}$ and y direction respectively. In general, Eq. (9) is computed by the fast Fourier transform (FFT) algorithm.

Combing Eq. (9) and Eq. (6) in Ref. [1], the deformed surface roughness $r r_{q_{x}, q_{y}}^{d}$ in the frequency domain is:

$r r_{q_{x}, q_{y}}^{d}=\left(\frac{A_{d}}{A_{i}}\right)_{q_{x}, q_{y}} \cdot\left|r r_{q_{x}, q_{y}}\right|$

According to the relation between the pressure and the elastic deformation of the waviness given in Ref. [32], the pressure increase in the frequency domain follows the expression below

$\delta p_{q_{x}, q_{y}}=\frac{\pi E^{\prime}}{\sqrt{2} \lambda}\left(r r_{q_{x}, q_{y}}-r r_{q_{x}, q_{y}}^{d}\right)$

Where $\lambda$ is defined as $\lambda=2 \pi \sqrt{q_{x}^{2}+q_{y}^{2}}$ in terms of the isotropic surface topography.

With the inverse discrete Fourier transform, the pressure increase in the space domain is obtained:

$\delta p_{x, y}=\frac{4}{N_{x} N_{y}} \sum_{q_{x}, q_{y}} \delta p_{q_{x}, q_{y}} \cdot e^{i\left(q_{x} x+q_{y} y\right)}$

According to Eq. (4), the ratio of the shear stress $\tau_{r} / \tau_{s}$ can be derived as:

$\frac{\tau_{r}(x, y)}{\tau_{s}(x, y)}=\frac{\eta_{r}(x, y)}{\eta_{s}(x, y)} \cdot \frac{h_{s}(x, y)}{h_{r}(x, y)}=\frac{\eta_{r}(x, y)}{\eta_{s}(x, y)} \cdot \frac{h_{s}(x, y)}{h_{s}(x, y)-a_{d}(x, y)}$

It is easy to obtain the shear stress distribution $\tau_{s}(x, y)$ or the smooth surface case, where the pressure distribution for the smooth surface case can be replaced by a semi-elliptical pressure distribution:

$p_{s}(x, y)=\left\{\begin{array}{cl}p_{h} \sqrt{1-\left(x / a_{h}\right)^{2}-\left(y / a_{h}\right)^{2}} & \text { if } x^{2}+y^{2} \leq a_{h}^{2} \\ 0 & \text { otherwise. }\end{array}\right.$ 
Afterwards, the pressure distribution for roughness cases is computed by $p_{s}+\delta p$. The shear stress distribution $\tau_{r}(x, y)$ for a rough surface case is obtained as:

$\tau_{r}(x, y)=\frac{\eta_{r}(x, y)}{\eta_{s}(x, y)} \cdot \frac{h_{s}(x, y)}{h_{s}(x, y)-a_{d}(x, y)} \cdot \tau_{s}(x, y)$

Finally, the shear forces for both of the smooth case and the rough case are computed by integrating the shear stress $\tau_{s}(x, y)$ and $\tau_{r}(x, y)$, respectively. The relative friction coefficient is then calculated according to Eq. (6). A detailed description for the prediction process of the relative friction coefficient is shown as Fig. 1.

\section{Results}

The relative friction coefficient is predicted for a harmonic surface roughness and for artificial fractal surface roughness respectively. The model validation is presented in section 4.1. Subsequently, in section 4.2 and 4.3 , prediction results for two types of surface topography are discussed.

\subsection{PSD friction model validation}

To validate the model described in Section 3, the relative friction coefficient evaluated from a full numerical simulation is compared with that predicted by PSD under the same operating conditions. The numerical simulation takes place on a domain $-2.5 \leq X \leq 1.5$ and $-2.0 \leq Y \leq 2.0$ with $513 \times 513$ equal-spaced points. The time step is selected equal to the spatial mesh size, i.e. with $\Delta T=H X=H Y=0.0078125$. Meanwhile, the calculation starts with $X_{s t}=-2.5$ and the surface topography moves into the high pressure zone with the velocity of the rough surface $u_{1}$. The monitoring time should be long enough so that the 'steady oscillations' of the results occur. What we considered in the present work is the small-amplitude roughness so that a small slip parameter is selected i.e. $U_{\text {rat }}=1.01$. This small slip assumption allows us to use the numerical solver for pure rolling and the Amplitude Reduction Theory [1] in pure rolling as well, as shown by Ref. [33]. In this study, the rough surface topography used is isotropic. Studies on non-isotropic surfaces will be considered in future work.

An artificial fractal rough surface is chosen to validate the model
Table 1

Operating condition parameters.

\begin{tabular}{lll}
\hline Parameter & Value & Units \\
\hline$w$ & 600 & $\mathrm{~N}$ \\
$\bar{u}$ & 0.84 & $\mathrm{~m} / \mathrm{s}$ \\
$R$ & 0.018 & $\mathrm{~m}$ \\
$E^{\prime}$ & $2.26 \mathrm{e} 11$ & $\mathrm{~Pa}$ \\
$\alpha$ & $2.2 \mathrm{e}-8$ & $\mathrm{~Pa}^{-1}$ \\
$\eta_{0}$ & $40 \mathrm{e}-3$ & $\mathrm{~Pa}^{-}$ \\
$\sigma$ & $0.05 \mathrm{e}-6$ & $\mathrm{~m}$ \\
$L_{x}=L_{y}$ & $8.29 \mathrm{e}-4$ & $\mathrm{~m}$ \\
$q_{r}$ & 0 & $\mathrm{~m}^{-1}$ \\
$N_{x}=N_{y}$ & 256 & \\
Hurst exponent & 0.8 & \\
\hline
\end{tabular}

Table 2

Relative friction coefficients as a function of the mesh points for two prediction schemes.

\begin{tabular}{llll}
\hline Mesh points $N_{x} \times N_{y}$ & $H_{x}=H_{y}$ & $\mu_{r} / \mu_{s}(\mathrm{PSD})$ & $\mu_{r} / \mu_{s}(\mathrm{EHL})$ \\
\hline $257 \times 257$ & $1 / 64$ & 1.53 & 1.48 \\
$513 \times 513$ & $1 / 128$ & 1.53 & 1.51 \\
$1025 \times 1025$ & $1 / 256$ & 1.53 & 1.51 \\
\hline
\end{tabular}

mentioned in section 3 and the operating condition parameters are listed in Table 1.

Table 2 presents the friction ratio as a function of the mesh points for the two methods. The results predicted by two schemes are basically identical. The ratio of friction coefficients predicted by the PSD method changes slightly $(<0.085 \%)$ with decreasing mesh size. However, in the full numerical simulation, a large mesh size leads to a relative high deviation. This is because some high frequency components of the rough surface can not be represented on such a large mesh size correctly. In this article, the precision of the numerical results simulated by $513 \times 513$ mesh points is considered acceptable.

\subsection{The isotropic harmonic surface roughness}

The isotropic harmonic surface pattern employed in this article is the same as that in Ref. [1], i.e.:

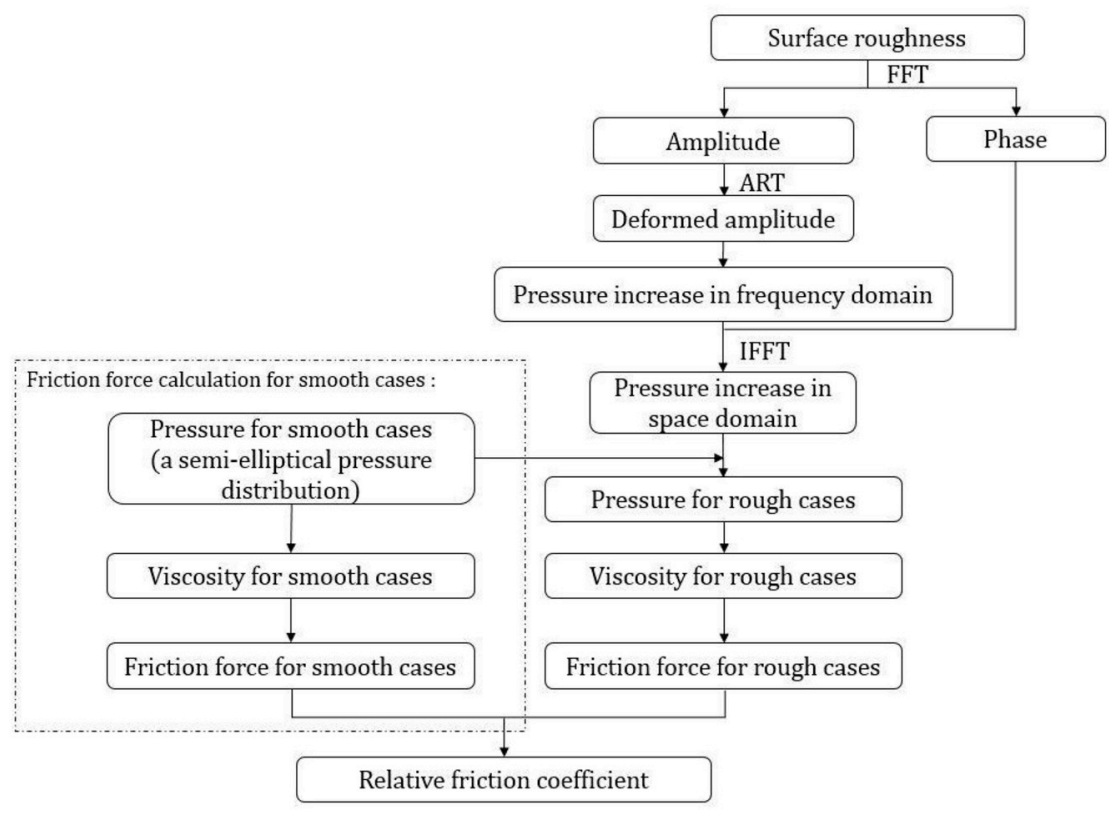

Fig. 1. Flow chart for the relative friction coefficient prediction. 


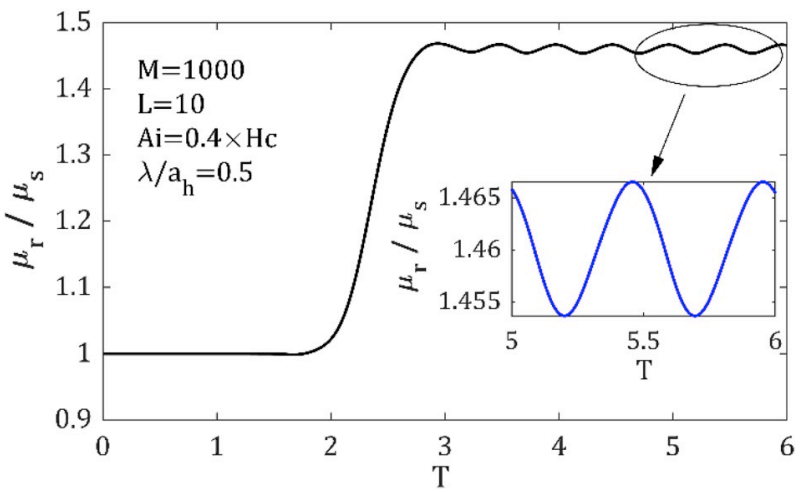

Fig. 2. The relative friction coefficient as a function of dimensionless time $\mathrm{T}$ for $M=1000, L=10, A_{i}=0.4 \times H_{c}$ and $\overline{\bar{\lambda}}=0.5$.

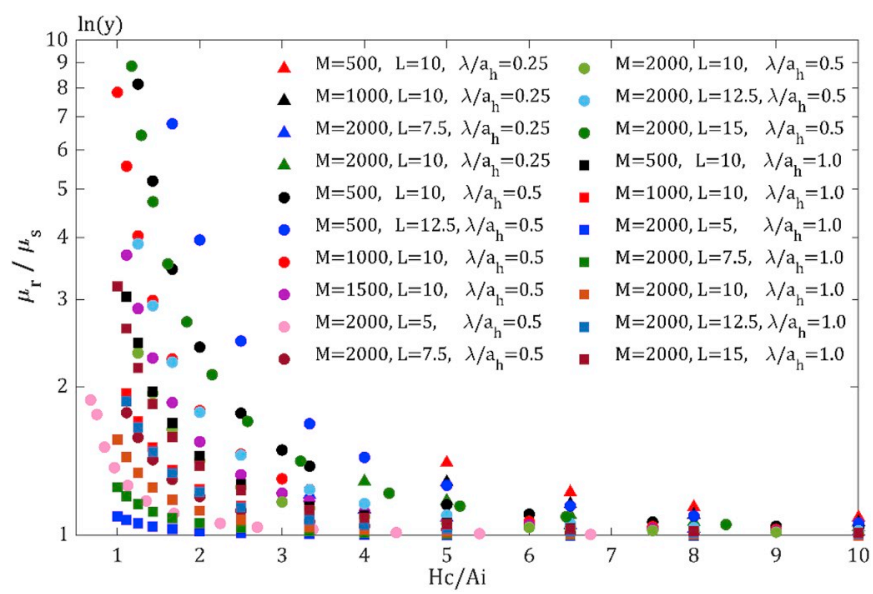

Fig. 3. The relative friction coefficient as a function of $H_{c} / A_{i}$.

$R R(X, Y, T)=A_{i} \cdot 10^{-10\left[\max (0,(X-X) / \overline{\bar{\lambda}})^{2}\right]} \cos \left(2 \pi \frac{X-\bar{X}}{\overline{\bar{\lambda}}}\right) \cos \left(2 \pi \frac{Y}{\overline{\bar{\lambda}}}\right)$

where $\bar{X}=X_{s t}+U_{r a t} \cdot T, A_{i}$ is the initial amplitude of the harmonic surface pattern, $\overline{\bar{\lambda}}$ is the dimensionless wavelength which equals to $\lambda_{x} / a_{h}$ or $\lambda_{y} / a_{h}$ for an isotropic surface pattern. The exponential term in Eq. (15) is used to avoid discontinuous derivatives when the roughness moves into the calculation domain.

Fig. 2 shows the relative friction coefficient as a function of the

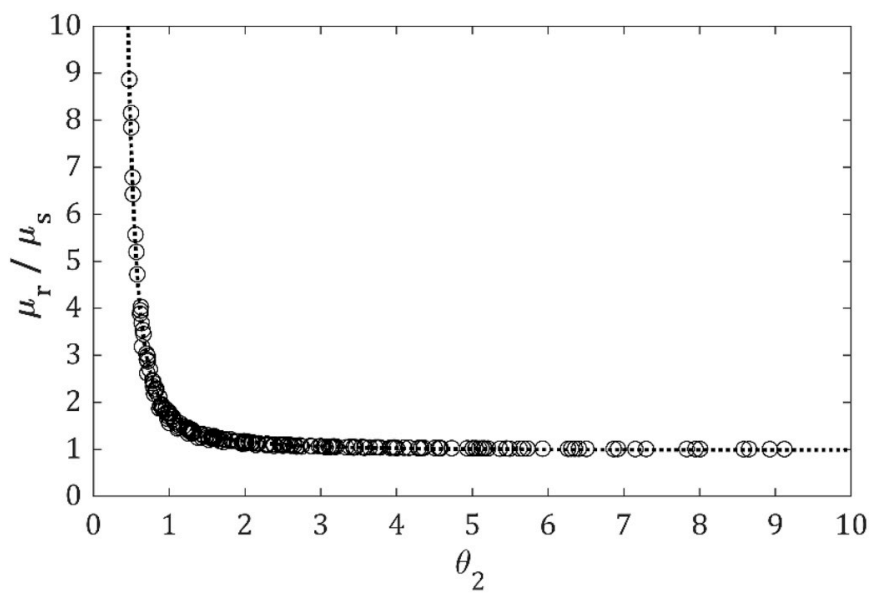

Fig. 4. The relative friction coefficient as a function of $\theta_{2}$.Dotted curve: equation (16). dimensionless time. The operating conditions are $M=1000, L=10$, $A_{i}=0.4 \times H_{c}=9.734 \times 10^{-3}$ and $\bar{\lambda}=0.5$. The value of $\mu_{r} / \mu_{s}$ is obtained by averaging $\mu_{r} / \mu_{s}(T)$ over a time period. For this case, the relative friction coefficients is $\mu_{r} / \mu_{s}=1.461$.

Fig. 3 presents the relative friction coefficient as a function of $H_{c} / A_{i}$ for many different operating conditions. It is readily observed that the relative friction coefficient decreases with increasing $H_{c} / A_{i}$. Indeed, with increasing $H_{c} / A_{i}$, the relative rough surface becomes 'smoother' and the relative friction coefficient approaches 1 . For each operating condition, a very smooth curve is obtained, however, we can not have a single curve like that for low pressure application through the parameter $H_{c} / A_{i}$ (or $h_{c} / \sigma$ ) to correctly describes the transition. According the Amplitude Reduction Theory [1], under very high pressure situation, surface roughness will be deformed. Instead of using this simple parameter $A_{i}$ or a measured surface roughness parameter $\sigma$, it is better to use the deformed parameter $A_{d}$.

Using the Amplitude Reduction Theory [1], it is possible to combine all results obtained for different values of $\overline{\bar{\lambda}}, M, L$ as well as $H_{c} / A_{i}$ into a single curve using a dimensionless parameter $\theta_{2}$. Fig. 4 shows the relative friction coefficient as a function of $\theta_{2}$ for $500 \leq M \leq 2000$, $5 \leq L \leq 15,0.25 \leq \overline{\bar{\lambda}} \leq 1.0$ and1.0 $\leq H_{c} / A_{i} \leq 10$. After curve-fitting, the single curve can be described by the following equation:

$\frac{\mu_{r}}{\mu_{s}}=1+0.546 \theta_{2}^{-2}+0.219 \theta_{2}^{-4}$

where $\theta_{2}=L^{-1.1} M^{0.33} \overline{\bar{\lambda}}^{0.67}\left(H_{c} / A_{i}\right)$.

The physical justification of this scaling parameter can be seen from a simplified analysis given in the Appendix.

\subsection{The artificial fractal surface roughness}

The artificial surface topography is generated by means of fractals. Fig. 5 depicts an artificial fractal surface topography and its corresponding "power spectral density" (PSD). This surface geometry is produced with the input parameters given in Table 1 without a roll-off region.

The resulting deformed micro-geometry, of which the original surface topography is shown in Fig. 5 (a) for a full numerical EHL simulation and a PSD prediction, are presented in Fig. 6. In terms of numerical simulation results, the deformed micro-geometry $A_{d}$ is obtained by $h_{s}-h_{r}$ and removing data outside the high-pressure zone. Once again, for this specific surface, the operating conditions are the ones given in Table 1 where $M=1000$ and $L=10$. Both the deformed surface topographies $A_{d}$ are shown in the same region $\left(X^{2}+Y^{2} \leq 1\right)$. The maximum Hertzian pressure reaches $1.66 \mathrm{GPa}$ and the maximum surface roughness height deformed significantly from $1 \times 10^{-7} \mathrm{~m}$ to $3.5 \times 10^{-8} \mathrm{~m}$. In addition, it is shown that the height distribution of the deformed surface roughness from the EHL simulation and the PSD prediction are very similar. The results of the numerical prediction are less detailed. This is because high frequency components of the surface roughness can not be well represented on the selected mesh. Therefore, these components are averaged.

Twenty artificial randomly rough surfaces were generated with the same input parameters i.e. the standard deviation $\sigma=5 \times 10^{-8} \mathrm{~m}$, lengths of final topography $L_{x}=L_{y}=8.29 \times 10^{-4} \mathrm{~m}$, roll-off wave number $q_{r}=0 \mathrm{~m}^{-1}$ and Hurst exponent $=0.8$. The relative friction coefficient values for these artificial randomly rough surfaces are given in Fig. 7, showing that the two different prediction methods give closer results. And the average deviation around $8 \%$.

\section{Conclusions}

An extended multigrid code incorporating Alcouffe's method [30] is applied in this paper. This numerical simulation tool is employed to generate the Stribeck curve in the full-film EHL regime. The Amplitude Reduction Theory is used to predict pressure increase due to waviness 


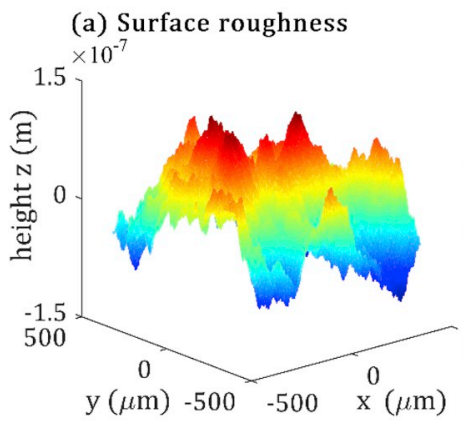

(b) 2D PSD

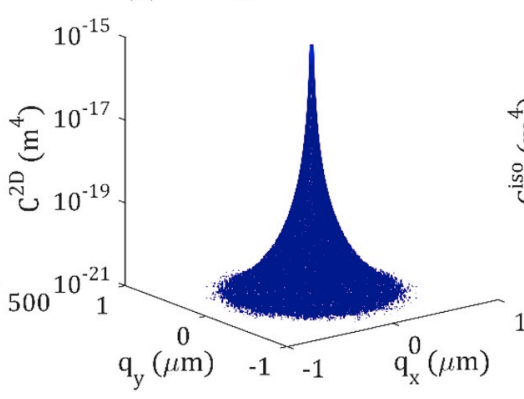

(c) Radially averaged

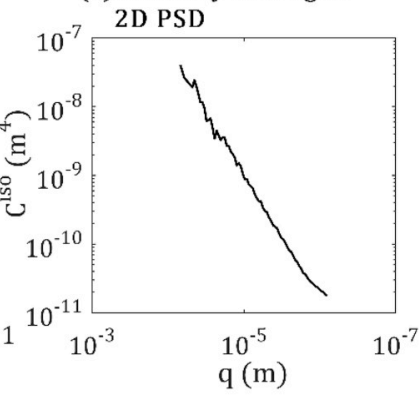

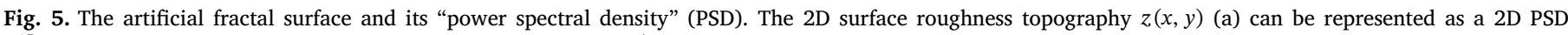
$C^{2 D}\left(q_{x}, q_{y}\right)(\mathrm{b})$, and for this isotropic surface, the radial average $C^{i s o}(q)$ is shown in (c).
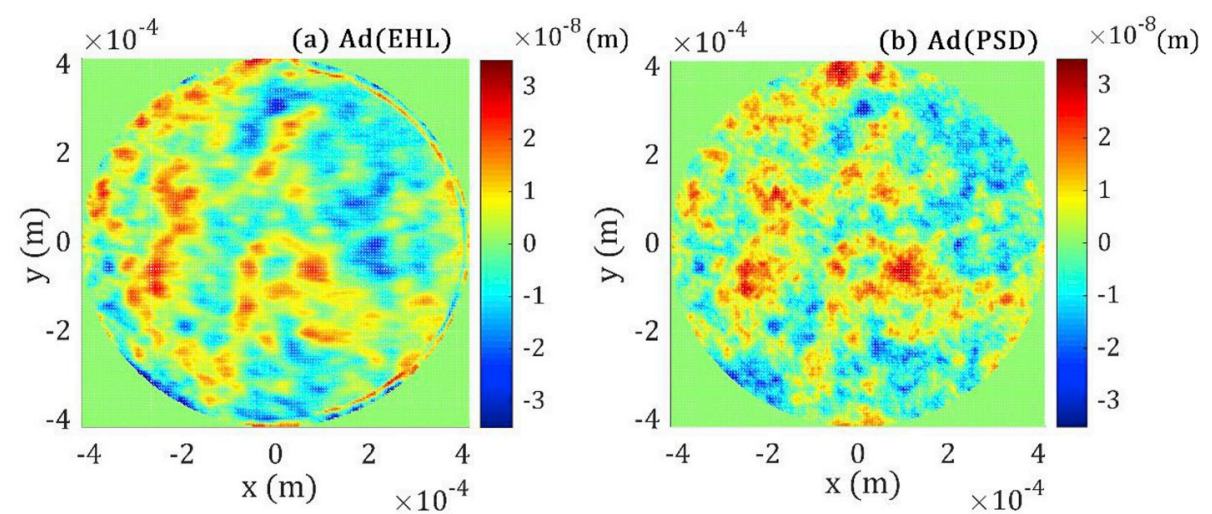

Fig. 6. Deformed surface roughness for a specific time step. Top view of the deformed surface roughness for a full numerical simulation (left) and for a PSD prediction (right).

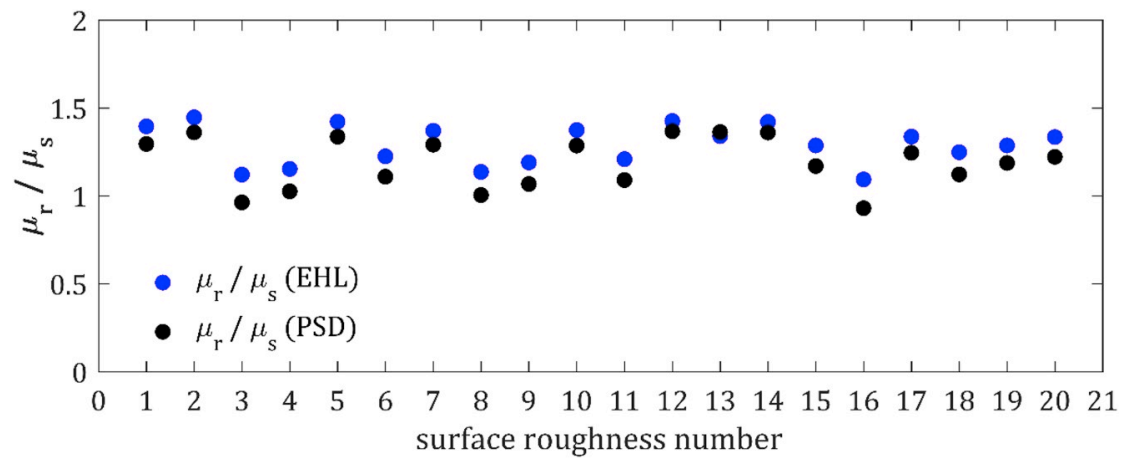

Fig. 7. The relative friction coefficient obtained by EHL simulation and PSD prediction for 20 artificial randomly rough isotropic surfaces.

deformation. This local pressure increase causes a friction increase. For many isotropic harmonic surfaces, all the relative friction coefficients fall onto a single curve using the dimensionless coordinate $\theta_{2}=L^{-1.1} M^{0.33} \overline{\bar{\lambda}}^{0.67}\left(H_{c} / A_{i}\right)$. This means that the transition from the mixed to the full-film regime is determined by $\theta_{2}$ and not simply by the lambda ratio. For a complex rough surface, thousands of time steps are needed for the full numerical simulation, which requires 3 days of computation. In this work, a rapid analytical prediction method, whose calculation time for each time step is only $2 \mathrm{~s}$ is proposed. The two methods show good agreement.

\section{Acknowledgment}

The first author would like to thank the China Scholarship Council (CSC) for its financial support.

\section{Appendix}

According to the Barus [34] viscosity-pressure equation, the shear stress ratio can be approximated as:

$\frac{\tau_{r}}{\tau_{s}} \approx e^{\bar{\alpha} \cdot \Delta P}=1+\bar{\alpha} \cdot \Delta P+\frac{(\bar{\alpha} \cdot \Delta P)^{2}}{2 !}+\frac{(\bar{\alpha} \cdot \Delta P)^{3}}{3 !}+\frac{(\bar{\alpha} \cdot \Delta P)^{4}}{4 !}+\ldots$ 
where the pressure increase $\Delta P$ has a linear relation with deformation [32], i.e. $\Delta P=\frac{\pi^{2} A_{i}}{2 \overline{\bar{\lambda}}}\left(1-\frac{A_{d}}{A_{i}}\right)$. Using a first order approximation of the dimensionless pressure increase $\Delta P, \bar{\alpha} \cdot \Delta P$ reduces to:

$\bar{\alpha} \cdot \Delta P \approx \bar{\alpha} \frac{\pi^{2} A_{i}}{2 \overline{\bar{\lambda}}} \approx\left[\frac{L}{\pi}\left(\frac{3 M}{2}\right)^{1 / 3}\right] \frac{\pi^{2} H_{c}}{2 \overline{\bar{\lambda}}}\left(\frac{H_{c}}{A_{i}}\right)^{-1}$

where $\bar{\alpha}$ is expressed as $\bar{\alpha}=\frac{L}{\pi}\left(\frac{3 M}{2}\right)^{1 / 3}$. Defining $H_{c}^{D}$ the dimensionless film thickness value using the well-known Hamrock-Dowson equation [35], $H_{c}^{D}=1.2 L^{0.53} U^{0.49} M^{-0.067}$. Now the dimensionless central film thickness $H_{c}$ can be rewritten as

$H_{c}=\frac{R_{x}^{2}}{a_{h}^{2}} \cdot H_{c}^{D}$

in which $R_{x}^{2} / a_{h}^{2}$ is expressed as $R_{x}^{2} / a_{h}^{2}=(3 / 2)^{-2 / 3} M^{-2 / 3} U^{-0.5}$.

Substituting Eq. (19) into Eq. (18) gives:

$\bar{\alpha} \cdot \Delta P \approx 1.6467\left[L^{-1.53} M^{0.4}(\overline{\bar{\lambda}})^{1}\left(H_{c} / A_{i}\right)\right]^{-1}$

Applying a second order approximation of $\Delta P, \bar{\alpha} \cdot \Delta P$ yields:

$\bar{\alpha} \cdot \Delta P \approx \frac{\pi^{2} A_{i}}{2 \overline{\bar{\lambda}}}\left(1-\frac{A_{d}}{A_{i}}\right) \approx 0.24\left[L^{-1.03} M^{-0.1}(\overline{\bar{\lambda}})^{0}\left(H_{c} / A_{i}\right)\right]^{-1}$

where $A_{d} / A_{i} \approx 1-0.15 \nabla_{2} \approx 1-0.15 \overline{\bar{\lambda}}(M / L)^{0.5}$.

Observing Eq. (20) and Eq. (21), the exponent of the parameter $M$ varies from -0.1 to 0.4 , the exponent of $L$ varies from -1.03 to -1.53 and that of $\overline{\bar{\lambda}}$ varies from 0 to 1 . Hence the expression of the $\theta_{2}$ parameter using $M^{0.33}, L^{-1.1}$ and $\overline{\bar{\lambda}}^{0.67}$ employs coefficients that fall in the range outlined above.

\section{References}

[1] Venner CH, Lubrecht AA. Amplitude reduction of non-isotropic harmonic patterns in circular EHL contacts, under pure rolling. Tribol 1999;36:151-62.

[2] Hersey MD. The laws of lubrication of horizontal journal bearings. J Wash Acad Sci 1914;4(19):542-52.

[3] Thurston RH. Friction and lubrication: determinations of the laws and coëfficients of friction by new methods and with new apparatus. Railroad gazette; 1879.

[4] Dowson D. History of tribology. Addison-Wesley Longman Limited; 1979.

[5] Stribeck R. Die wesentlichen Eigenschaften der Gleit- und Rollenlager. Berlin: Springer; 1903.

[6] Gümbel L. Das problem der Lagerreibung vol. 5. Mbl Berl. Bez Ver Dtsch Ing; 1914.

[7] Wilson RE, Barnard DP. The mechanism of lubrication. Warrendale, PA: SAE International; Jan. 1922. SAE Technical Paper 220008.

[8] McKee SA. The effect of running-in on journal bearing performance. Mech Eng 1927;49:1335-40.

[9] Vogelpohl G. Die Stribeck-Kurve als Kennzeichen des allgemeinen Reibungsverhaltens geschmierter Gleitflächen. Z VDI 1954:96(9):261-8.

[10] Spikes HA. Mixed lubrication—an overview. Lubric Sci 1997;9(3):221-53.

[11] Shotter BA. Experiments with a disc machine to determine the possible influence of surface finish on gear tooth performance. Proc. Int. Conf. Gearing. vol. 120. 1958.

[12] Tallian TE, Chiu YP, Huttenlocher DF, Kamenshine JA, Sibley LB, Sindlinger NE. Lubricant films in rolling contact of rough surfaces. ASLE Trans. 1964;7(2):109-26.

[13] Tallian TE, McCool JI, Sibley LB. Paper 14: partial elastohydrodynamic lubrication in rolling contact. Proceedings of the Institution of Mechanical Engineers, Conference Proceedings, vol. 180. 1965. p. 169-86.

[14] Poon SY, Haines DJ. Third paper: frictional behaviour of lubricated rolling-contact elements. Proc Inst Mech Eng 1966;181(1):363-89.

[15] Bair S, Winer WO. Regimes of traction in concentrated contact lubrication. J Lubr Technol 1982;104(3):382-6.

[16] Stachowiak G, Batchelor AW. Engineering tribology. Butterworth-Heinemann; 2013.

[17] Cann P, Ioannides E, Jacobson B, Lubrecht AA. The lambda ratio-a critical reexamination. Wear 1994;175(1-2):177-88.

[18] Schipper DJ. Transitions in the lubrication of concentrated contacts PhD Thesis University of Twente; 1988
[19] Gelinck ERM, Schipper DJ. Calculation of Stribeck curves for line contacts. Tribol Int 2000;33(3-4):175-81.

[20] Johnson KL, Greenwood JA, Poon SY. A simple theory of asperity contact in elastohydro-dynamic lubrication. Wear 1972;19(1):91-108.

[21] Lu X, Khonsari MM, Gelinck ER. The Stribeck curve: experimental results and theoretical prediction. J Tribol 2006;128(4):789-94.

[22] Wang W, et al. Simulations and measurements of sliding friction between rough surfaces in point contacts: from EHL to boundary lubrication. J Tribol 2007;129(3):495-501.

[23] Kalin M, Velkavrh I, Vižintin J. The Stribeck curve and lubrication design for nonfully wetted surfaces. Wear 2009;267(5-8):1232-40.

[24] Kalin M, Velkavrh I. Non-conventional inverse-Stribeck-curve behaviour and other characteristics of DLC coatings in all lubrication regimes. Wear 2013;297(1-2):911-8.

[25] Zhang X, Li Z, Wang J. Friction prediction of rolling-sliding contact in mixed EHL. Measurement 2017;100:262-9.

[26] Bonaventure J, Cayer-Barrioz J, Mazuyer D. Transition between mixed lubrication and elastohydrodynamic lubrication with randomly rough surfaces. Tribol Lett 2016;64(3):44.

[27] Venner CH, Lubrecht AA. Multi-level methods in lubrication vol. 37. Elsevier; 2000.

[28] Dowson D, Higginson GR. Elastohydrodynamic lubrication, the fundamentals of roller and gear lubrication. Oxford: Pergamon; 1966.

[29] Roelands CJA. Correlational aspects of the viscosity-temperature-pressure relationship of lubricating oils PhD Thesis Delft: University of Delft; 1966

[30] Alcouffe R, Brandt A, Dendy J, Painter J. The multi-grid method for the diffusion equation with strongly discontinuous coefficients. SIAM J Sci Stat Comput Dec. 1981;2(4):430-54.

[31] Jacobs TDB, Junge T, Pastewka L. Quantitative characterization of surface topography using spectral analysis. Surf Topogr Metrol Prop 2017;5(1):013001.

[32] Johnson KL, Johnson KL. Contact mechanics. Cambridge University Press; 1987.

[33] Šperka P, Křupka I, Hartl M. Experimental study of real roughness attenuation in concentrated contacts. Tribol Int Oct. 2010;43(10):1893-901.

[34] Barus C. Isothermals, isopiestics and isometrics relative to viscosity. Am J Sci Feb. 1893;45(266):87-96. vol. Series 3.

[35] Hamrock BJ, Dowson D. Isothermal elastohydrodynamic lubrication of point contacts: Part 1-theoretical formulation. J Lubr Technol Apr. 1976;98(2):223-8. 